\title{
A test of the 1992 International Standards for Neurological and Functional Classification of Spinal Cord Injury
}

\author{
Michelle E Cohen ${ }^{1}$, John F Ditunno $\mathrm{Jr}^{1}$, William H Donovan ${ }^{2}$ and Frederick M Maynard $\mathrm{Jr}^{3}$ \\ ${ }^{1}$ Department of Rehabilitation Medicine, Jefferson Medical College, Thomas Jefferson University, Philadelphia, \\ Pennsylvania, USA; ${ }^{2}$ The Institute for Rehabilitation and Research, Houston, Texas, USA; ${ }^{3}$ Department of Physical \\ Medicine and Rehabilitation, MetroHealth Medical Center, Cleveland, Ohio, USA.
}

\begin{abstract}
This study was designed to test the 1992 International Standards for Neurological and Functional Classification of Spinal Cord Injury. One hundred and six professionals in the field of spinal cord injury attending an instructional course at the 1994 ASIA Meeting participated in the test. Participants completed a pretest and posttest in which they classified two patients who had a spinal cord injury (one with complete tetraplegia and one with incomplete paraplegia) by sensory and motor levels, zone of partial preservation (ZPP), ASIA Impairment Scale and completeness of injury. Between tests, three members of the ASIA Standards Executive Committee gave presentations on the neurological assessment, scoring, scaling and classification of spinal cord injury and a video of the actual examinations of the two cases was viewed. Percent 'correct' (as defined by the ASIA Standards Committee) was calculated for sensory and motor levels, ZPP, ASIA Impairment and completeness. Overall, the analyses showed that participants had very little difficulty in correctly classifying the patient with complete tetraplegia. Pretests scores ranged from $72 \%$ (left motor level) to $96 \%$ (complete injury), posttest scores from $73 \%$ (left motor level) to $100 \%$ correct (complete injury). For the patient with incomplete paraplegia (Case 2), scores were considerably lower. Pretest scores ranged from $16 \%$ (right motor level) to $95 \%$ correct (incomplete injury); posttest scores from $21 \%$ (right motor level) to $97 \%$ correct (incomplete injury). The results showed that further revisions of the 1992 Standards and more training is needed to ensure accurate classification of spinal cord injury.
\end{abstract}

Keywords: spinal cord injury; classification; ASIA/IMSOP standards

\section{Introduction}

The precise classification of spinal cord injury is essential for determining the severity of injury, prognosis, plan of rehabilitation, and the outcomes and benefits from interventions. The American Spinal Injury Association first published a standard system for the neurological classification of spinal cord injury in $1982^{1}$. The application of these standards require two different skills; the skill of examination and the skill of classification. The classification of injury is based on the data obtained from the neurological examination. Reliability in one skill does not guarantee reliability in the other. Further, it is important that procedures used for both examination and classification undergo testing to establish validity and reliability. Changes made to these procedures should be based on empirical evidence. Over the past 14 years, much progress has been made in refining the standards of classification. ${ }^{2}$ The changes that have been made thus far were primarily based on research findings.

Correspondence: M Cohen, Department of Rehabilitation Medicine, Thomas Jefferson University, 1015 Walnut Street, Suite 617, Philadelphia, PA 19107, USA
In 1988, Donovan and his colleagues ${ }^{3}$ tested the classification skill of a group of experts using the 1982 ASIA Standards. ${ }^{1}$ The results showed a great deal of disparity existed, even among these 'experts', in classifying the motor levels, sensory levels and Frankel grades. In order to improve the problems in neurological classification, the ASIA Standards were revised in 1989. The revisions included clarification of muscle grading used in the determination of motor levels, the inclusion of anatomical landmarks within the dermatomes to define sensory levels, redefinition of the zone of partial preservation for motor and sensory function and clarification of the Frankel grading system, as recommended by Tator, ${ }^{4}$ to more precisely determine the degree of incompleteness.

Priebe and Waring ${ }^{5}$ compared the original 1982 Standards to the 1989 revisions. Although there was improvement with the 1989 revisions, agreement among the 14 physicians who performed the classifications was only fair. These findings suggested that further revisions were necessary to obtain optimal agreement. Priebe and Waring suggested that the use of key sensory areas was important for the dermatome 
chart and that there should be further clarification in the classification of incomplete injuries. The investigators went on to suggest that training methods be developed for the ASIA Standards.

In 1992, major revisions were made to the standards as a result of the methylprednisolone study ${ }^{6}$ and to address the problems presented by Priebe and Waring. ${ }^{5}$ With these revisions, the Frankel grading system was further refined and is now called the ASIA Impairment Scale (AIS). This third revision of the American Spinal Injury Association (ASIA) Standards has been endorsed by the International Medical Society of Paraplegia (IMSOP). ${ }^{7}$ The international community of clinicians and researchers in spinal cord injury now has a common system for accurate communication. As a result of IMSOP's endorsement, the Standards are now known as the International Standards for Neurological and Functional Classification of Spinal Cord Injury (ISCSCI). In addition, a training package for the Standards was developed in 1994 which included a reference manual and four video tapes. ${ }^{8}$

The previous evaluations of the Standards tested only the skill of classification. For the first time, Cohen and Bartko 9 tested both interrater and intrarater reliability for the skills of examination and classification, using the 1992 Standards. Twenty-nine clinicians from 19 medical institutions in the US and Canada examined and classified 32 individuals with spinal cord injury. The raters received training in the neurological examination procedures but not in the classification system. The changes made in 1992 resulted in excellent reliability for the examination procedures but revealed that discrepancies still existed in the classification of injury. ${ }^{9,10}$ The findings concluded that the skill of classification is not only based on the clinical data provided by the neurological examination but also on training.

The present study was designed to test the effect of training in a conference setting on the skills of classification. The study was conducted in 1994 to test the ISCSCI-92 classification system and the training procedures developed for this system.

\section{Methods}

The study was conducted at an instructional course presented at the 1994 ASIA Annual Meeting. One hundred and twenty-five participants attended the instructional course. Data from 106 of the participants who completed both a pretest and posttest was analyzed for this paper. Thirty-nine of these participants were physicians, 31 physical therapists, 15 occupational therapist, 15 nurses and six classified as other rehabilitation professionals. Twenty-seven percent of the participants had 1 year or less experience in spinal cord injury, $35 \%$ had 2 to 5 years experience, $22 \%$ had 6 to 10 years and $17 \%$ had more than 10 years of experience.

Upon entering the instructional course, each participant was given a packet containing a colorcoded and numbered pretest and posttest and a written description of two cases of spinal cord injury. Each test had a pressure sensitive copy attached so that participants could keep a copy of their answers. Participants were asked to complete the pretest immediately and all pretests were collected prior to the start of the course. Both tests consisted of classifying the two written cases of spinal cord injury; one with complete tetraplegia and the other with incomplete paraplegia. Classification consisted of determining the motor and sensory neurological levels, the zones of partial preservation, the ASIA Impairment Scale (AIS) and whether the injury was complete or incomplete. The cases were taken from the Reference Manual for the International Standards for Neurological and Functional Classification of Spinal Cord Injury. ${ }^{8}$ Following the pretest, participants viewed the $25 \mathrm{~min}$ ASIA training video which presented the actual neurological examinations of the two written cases and listened to $20 \mathrm{~min}$ presentations by Drs Maynard, Donovan and Ditunno which explained the general procedures for classification of spinal cord injury. The presentations were followed by the posttest, which was identical to the pretest. The posttests were collected and discussion of the answers followed. Below are the actual case reports the subjects were given for classification.

\section{Case reports}

Case 1 Table 1 presents the motor and sensory information obtained from the neurological examination performed on an individual with a complete tetraplegic injury. This patient had normal strength (grade 5) in the elbow flexors (C5). The wrist extensors on the right side had normal strength (grade 5). However, the left wrist extensor was weak and provides only moderate resistance (grade 4). The elbow extensors (C7) were weaker; left side moved through the defined range of motion, with gravity eliminated (grade 2), but the right side was unable to move through the defined range (grade 1). No voluntary movement was found in the finger flexors or small finger abductors. This was also the case in the lower extremities. The total motor score for this patient was 22. The sensory findings were symmetrical for light touch and pin prick. $\mathrm{C} 2-\mathrm{C} 5$ was normal for both testing modalities, but impaired for both in the C6 dermatome, on both sides. No sensation was found distal to C6. Consequently, the sensory scores were 18 for light touch and also 18 for pin prick.

Case 2 Table 2 presents the information obtained from the neurological examination on an individual with incomplete paraplegia. In this patient, the key muscles of the upper extremity tested normal on each side; therefore, C5-T1 key muscles each received a grade of 5 , for a score of 25 for each side. The muscles of the lower extremities showed asymmetry. Hip flexors (L2) on the right and left side were able to give normal resistance and received a grade of 5 . The right knee extensor (L3) also gave normal resistance, receiving a 
Table 1 Case 1, neurological examination

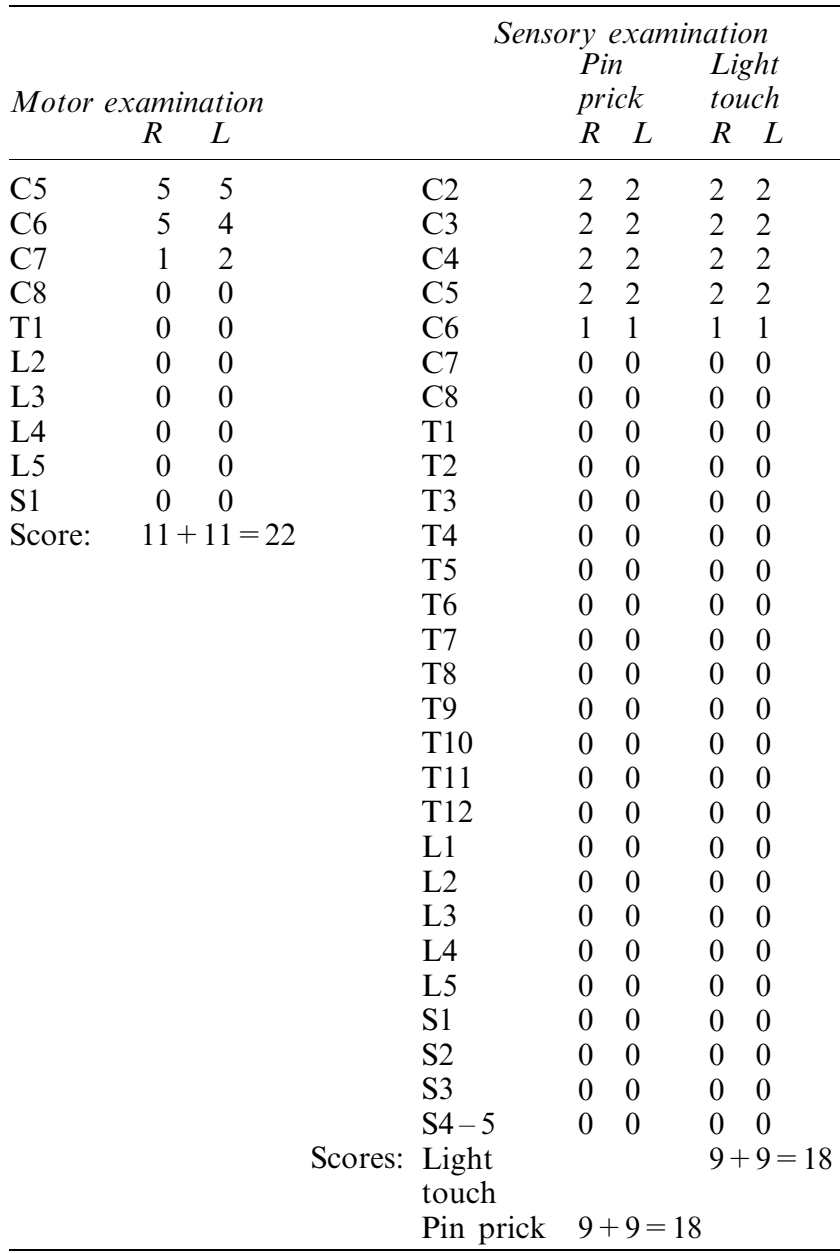

Data obtained from the neurological examination of Case 1. Muscle grades for $10 \mathrm{key}$ muscles on the right and left sides of the body. Pin prick and light touch scores for the 28 dermatomes on the right and left side of the body.

grade of 5; the left side could only go through a range of motion with gravity eliminated (grade 2). Ankle dorsiflexors (L4) were weak; the right side provided moderate resistance (grade 4), while the left side extended against gravity (grade 3 ). Long toe extensors (L5) gave resistance against gravity and received a grade of 3. Ankle plantar flexors (S1) did not provide resistance against gravity (by lifting the heel completely off the bed), whereas they could flex the ankle when gravity was eliminated (grade 2). The motor score was calculated by adding the grades of each muscle tested, achieving a total of 84 (50 for upper extremities, plus 19 lower right and 15 for lower left).

With this patient, the scoring for the light touch was different from that for pin prick. Light touch sensation was preserved to some extent in all dermatomes $(\mathrm{C} 2-$ S4-5). Multiple dermatomes below T7 were hyperesthetic, but the patient could not distinguish between pin prick and dull sensation, and was consequently graded 0 in those dermatomes. For calculating the
Table 2 Case 2, neurological examination

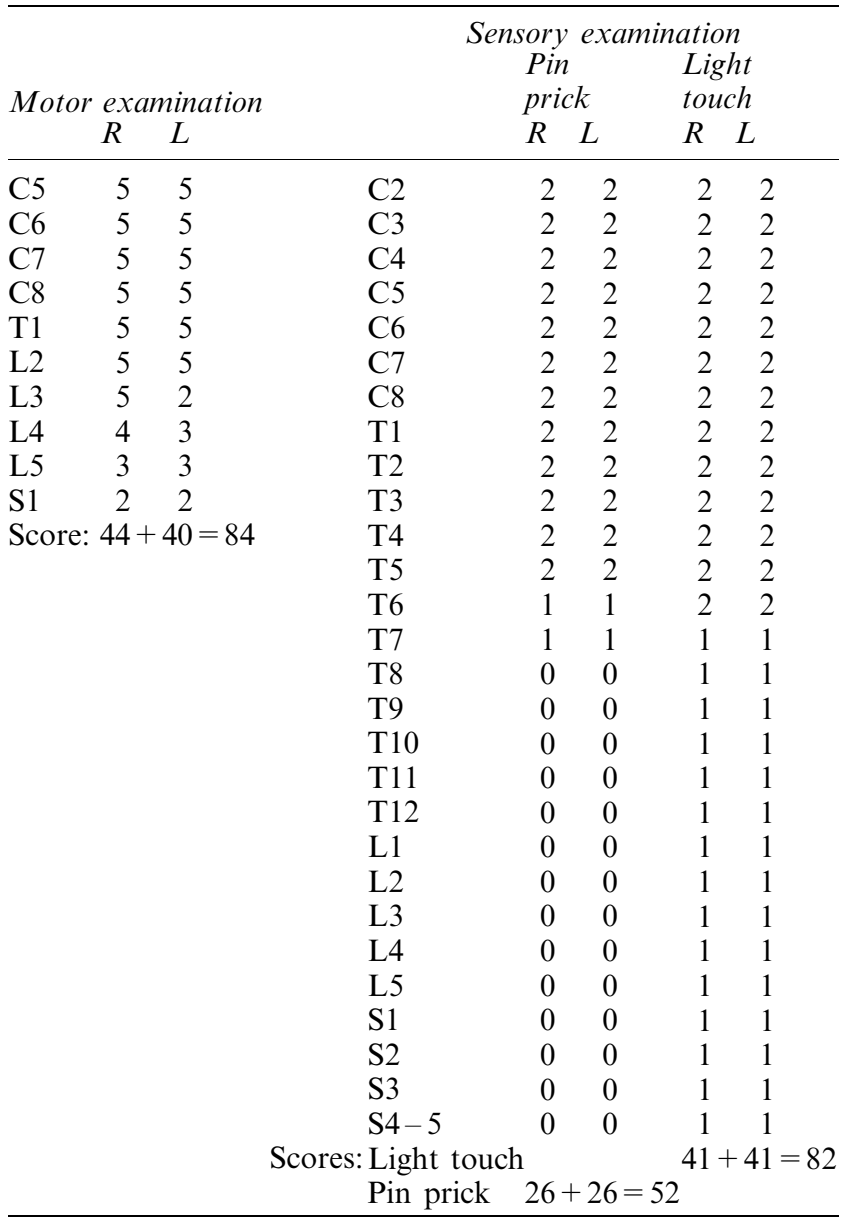

Data obtained from the neurological examination of Case 2. Muscle grades for 10 key muscles on the right and left sides of the body. Pin prick and light touch scores for the 28 dermatomes on the right and left side of the body.

light touch sensory score the findings were symmetrical, with normal sensation in 13 dermatomes $(\mathrm{C} 2-$ T6), and impaired light touch sensation from $\mathrm{T} 7$ through S4-5 (15 dermatomes). The score for each side was 41 , with a total light touch sensory score of 82. In calculating the pin prick sensory score, the findings were also symmetrical, with normal sensation in 12 dermatomes $(\mathrm{C} 2-\mathrm{T} 5)$ and impaired pin prick sensation in two dermatomes (T6 and T7). The 14 dermatomes distal to $\mathrm{T} 7$ were graded as 0 , as the patient could not distinguish dull from sharp. The total pin prick sensory score was 52 .

\section{Results}

Data were analyzed by calculating the percent 'correct' (defined by the ASIA Standard's Committee ${ }^{8}$ ) for right and left sensory and motor levels, zones of partial preservation (ZPP), AIS grade and identifying the injury as complete or incomplete. 
Case 1 Table 3 shows the percent correct for the pretest and posttest for Case 1. Case 1 was an example of complete (AIS grade A) tetraplegia with motor level C6 bilaterally, sensory level C5 bilaterally, ZPPs were to $\mathrm{C} 7$ motor and to C6 sensory bilaterally.

Chi Square analyses showed no significant differences between the pretest and posttest on any of the classification variables. More than $90 \%$ of the participants correctly classified the right motor level, both sensory levels, ZPPs and AIS grade and completeness of injury. Classification of the left motor level, however, caused problems for more than $25 \%$ of the participants. Furthermore, after the training only one participant changed to the correct answer.

When looking at these results in more detail, Figure 1 shows that approximately $23 \%$ of the participants incorrectly classified the left motor level as C5 on both the pretest and posttest. These mistakes resulted from confusion on how to classify the motor level when a muscle is a grade 4 with the next most rostral muscle a grade 5 . In this case, the C6 muscle was a grade 4 and C5 muscle a grade 5 .

Table 3 Percentage of correct classifications for Case 1 on the Pretest and Posttest

\begin{tabular}{lcc}
\hline Variable & Pretest & Posttest \\
\hline Neurological levels & & \\
$\quad$ Right motor level & 92 & 97 \\
Left motor level & 71 & 73 \\
Right sensory level & 92 & 95 \\
Left sensory level & 91 & 95 \\
Zones of partial preservation & & \\
Right motor ZPP & 95 & 92 \\
Left motor ZPP & 94 & 90 \\
Right sensory ZPP & 93 & 93 \\
Left sensory ZPP & 91 & 91 \\
ASIA Impairment Scale (AIS) & 94 & 98 \\
Complete injury & 96 & 100 \\
\hline
\end{tabular}

\section{CASE 1}

Motor Level Classifications

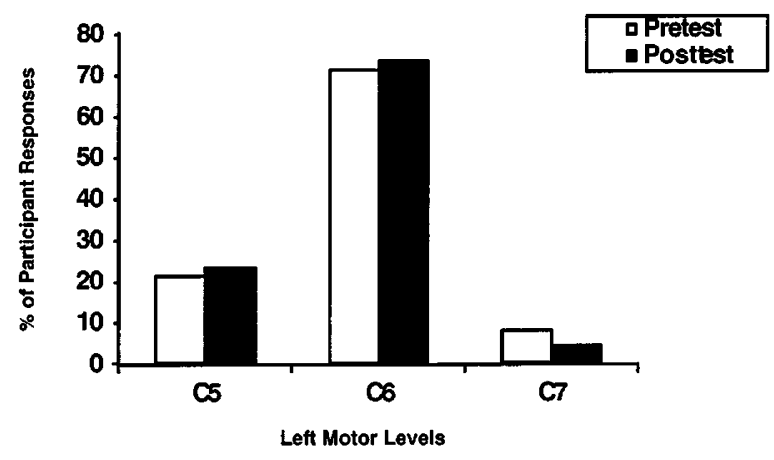

Figure 1 Participants' responses for the classifications of the left motor level in Case 1 on the pretest and posttest. The correct classification was $\mathrm{C} 6$
Case 2 Table 4 shows the percentage of correct answers for Case 2. Case 2 was an example of incomplete (AIS grade D) paraplegia with motor level L4 on the right side and L2 on the left side. Sensory levels were at T5 bilaterally. Because Case 2 was an incomplete injury, the ZPPs were not applicable to this case.

It is apparent from the results that the participants had more difficulty when classifying Case 2, the more complicated case of an asymmetrical incomplete paraplegic injury. The training received in the instructional course, although limited, resulted in statistically significant improvement in the understanding of the concept of the ZPP. However, approximately $35 \%$ of the participants were still confused following the training and delineated a classification of the ZPP which was incorrect for incomplete injuries.

While there was some improvement in the classification of the AIS, the improvement after training was not sufficient to show a significant training effect. The limited training also had no impact on classification of the neurological levels for Case 2. Significant problems still occurred in classifying both the motor and sensory levels. Figure 2 shows the results for classification of the right motor level. Only $21 \%$ of the participants classified the right motor level as L4, the answer given by the ASIA Standards Committee. An equal number $(21 \%)$ classified the right motor level as L3 and more than half of the participants $(56 \%)$ classified the right motor level as L5. The problem again appeared to be caused by confusion with classifying the level when there is a muscle of grade 4 (L4), the muscle immediately above it is grade 5 (L3) and the muscle below (L3) is a grade 3.

Sensory classification also caused some problems. Figure 3 shows the sensory classifications for the right and left sides on the posttest. Of those who incorrectly classified the sensory levels $(13 \%$ for the right side and $32 \%$ for the left side), most classified the level as T6. Light touch was normal bilaterally at T6 but pin was

Table 4 Percentage of correct classifications for Case 2

\begin{tabular}{lcccc}
\hline Variable & \multicolumn{5}{c}{ Chi } \\
Pretest & Posttest & square & $\mathrm{P}$ \\
\hline Neurological levels & & & & \\
$\quad$ Right motor level & 16 & 21 & $<1$ & $\mathrm{NS}$ \\
$\quad$ Left motor level & 87 & 84 & $<1$ & $\mathrm{NS}$ \\
$\quad$ Right sensory level & 87 & 87 & $<1$ & $\mathrm{NS}$ \\
$\quad$ Left sensory level & 69 & 68 & $<1$ & $\mathrm{NS}$ \\
Zones of partial preservation & & & & \\
$\quad$ Right motor ZPP & 19 & 66 & 24.89 & $<0.001$ \\
$\quad$ Left motor ZPP & 20 & 65 & 22.78 & $<0.001$ \\
Right sensory ZPP & 19 & 65 & 25.49 & $<0.001$ \\
$\quad$ Left sensory ZPP & 20 & 65 & 24.89 & $<0.001$ \\
ASIA Impairment Scale (AIS) & 58 & 65 & $<1$ & $\mathrm{NS}$ \\
Incomplete injury & 95 & 97 & $<1$ & $\mathrm{NS}$ \\
\hline
\end{tabular}




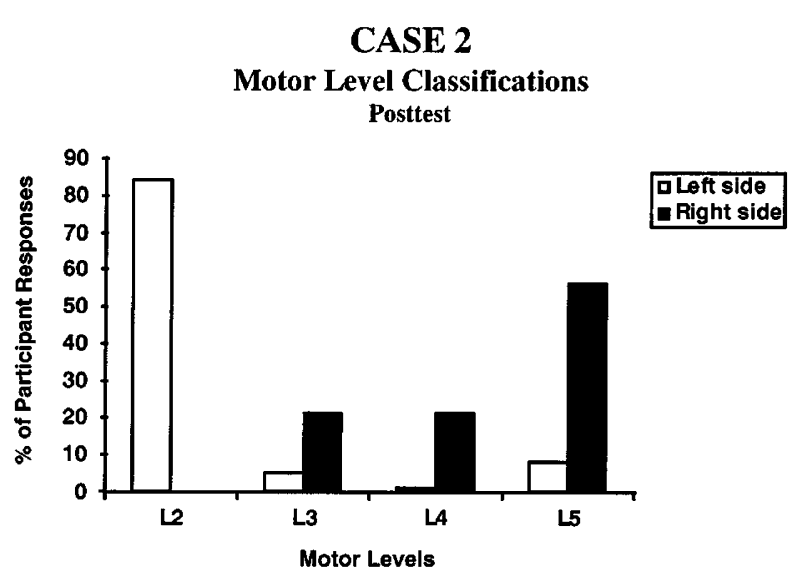

Figure 2 Participants' responses on the posttest classification of the right and left motor levels for Case 2. The correct classification was L4 right motor level and L2 left motor level

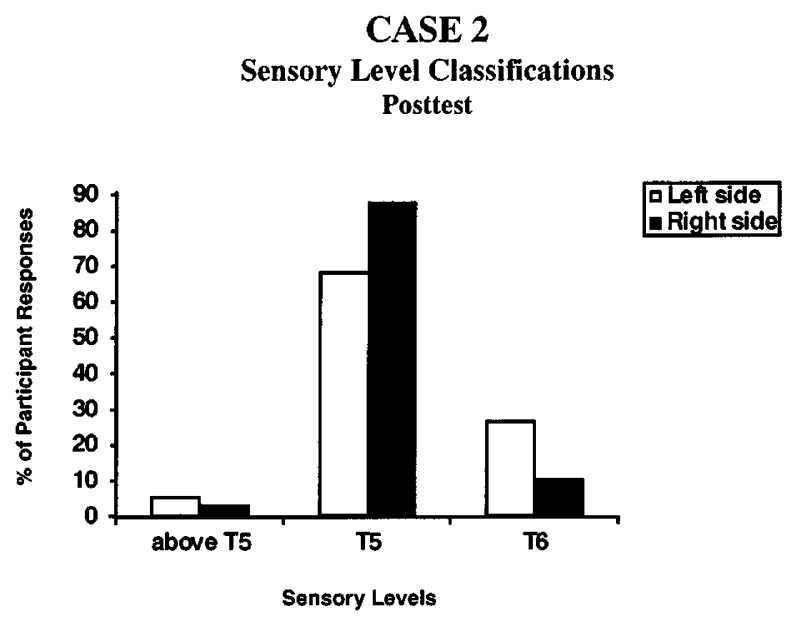

Figure 3 Participants' responses on the posttest classification of the right and left sensory levels for Case 2. The correct classification was T5 bilaterally

impaired at this level. To correctly classify the sensory level, both pin and light touch must be normal (score of 2).

Data from each case was further analyzed by professional background (physician, nurse, PT, OT or other) and years of experience. Neither of these variables yielded any differences from the overall group data for Case 1 or Case 2.

\section{Discussion}

The results of this study suggest that classification of complete injuries, such as in Case 1, is relatively easy. Little training is necessary for classifying this type of injury. For Case 1, the amount of training received in the instructional course produced only small improvement in classifications that were already correct by at least $90 \%$ of the participants.

In both the complete and incomplete cases problems still occurred following training for the classification of motor levels when muscles of grade 4 are present. As a result of preliminary analyses, this problem was recently addressed by the ASIA Standards Committee and further revisions have been made to the classification of motor levels. ${ }^{11}$ The statement on motor level classification was reworded and now reads '. . . , the motor level ... is defined by the lowest key muscle that has a grade of at least 3, providing the key muscles represented by segments above that level are judged to be normal (5).' Previously, this statement read that normal could be 4 or $5 .^{12}$ By clarifying the motor grade necessary for a normal muscle, the latest revision should eliminate the problems which occurred in classification of the motor levels in this study and which may explain why participants did not improve even after training.

Classification of the incomplete injury, Case 2, presented in this study was problematical in many areas. In addition to the motor level classification, sensory level classification, determination of the ZPP and AIS classification were still confusing for many of the participants even after the training session.

In determination at the sensory level, both the right and left sensory levels were T5, the most caudal level at which both light touch and pin prick were normal (two). Those participants who misclassified the sensory level chose T6, where light touch was normal (two) but pin prick was impaired (one). This mistake may be corrected with more extensive training and usage of the classification system. Further, the Standards booklet ${ }^{11}$ should reiterate the proper classification of sensory level in the discussion of sensory scores and sensory level (p15). Sensory classification is discussed under the definitions (p6) but not in the section that describes the sensory level (p15). By presenting the discussion on proper classification of the sensory level in more than one area in the Standards booklet, the reader's learning should be reinforced.

Further, there was a large discrepancy between the classification of the right and left sensory levels. Eighty-seven percent of the participants correctly classified the right sensory level as T5, while only $69 \%$ correctly classified the left sensory level as T5. This discrepancy could have been the result of the tabular presentation of the cases (see Table 2). The sensory information was presented with the right and left side values as subheadings under the headings for pin prick and light touch. In some institutions, forms used to document the neurological examination are set up so that pin prick and light touch values are subheadings under 'right side' and 'left side'. Individuals misreading the headings as the latter setup would erroneously classify the sensory level as T5 on the right and T6 on the left. This is a possible 
explanation for the $18 \%$ difference in the number of correct classifications for the right and left sensory levels.

The problem with determination of the ZPP for Case 2 occurred when the participants failed to realize that the term ZPP is only used for complete injuries. Following training, $97 \%$ of the participants correctly classified the case as an incomplete injury, but only $65 \%$ correctly classified the ZPP as not applicable for this case. Again, further training and usage of the Standards may correct this problem. More discussion in the Standards booklet and manual hopefully will help to prevent this mistake.

Finally, after training only $65 \%$ of the participants were able to correctly determine that the AIS was D Incomplete. Those $35 \%$ of the participants who incorrectly classified the Impairment chose C Incomplete. This may have been due to the wording of the definitions for $\mathrm{C}$ and $\mathrm{D}$ Incomplete injuries. In the 1992 Standards booklet (ASIA, 1992), the definitions read that for classification of $\mathrm{D}$ the 'majority of key muscles below the neurological level have a muscle grade greater than or equal to 3'; while for a classification of $\mathrm{C}$ 'the majority of muscles below the neurological level have a muscle grade less than three'. The term majority may have caused this confusion in the classification. This problem was initially addressed in the 1994 Reference Manual and has now been incorporated into 1996 revisions of the Standards. The term majority has been replaced with 'at least half of the key muscles' for the classification of D and 'more than half of the key muscles' for the classification of C. Empirical testing of the 1996 revisions will determine if these changes have corrected the problems in the classification of injuries.

There could be another possible explanation for the incorrect classification of the AIS. For the participants who used L4 right and L2 left as the motor levels, the AIS grade could be C or D based on the 1992 Standards. Because $50 \%$ of the muscles below the motor levels were less than grade 3, this could result in the classification of AIS C. But $50 \%$ of the muscles were also at least grade 3 ; therefore this qualifies as an AIS Grade D. For those participants who used L3 right and L2 left motor levels, the AIS grade would be D since four of seven key muscles were at least grade 3 . For those who chose L5 right and L2 left motor levels, the AIS grade $\mathrm{C}$ would result since three of five key muscles were less than grade 3. And for those participants who chose T5 bilaterally as the motor level, AIS D would result since seven out of ten key muscles are at least grade 3. The 1996 Standards now instruct the examiners that when the sensory level falls into a region where the muscles cannot be clinically graded $(\mathrm{C} 1-\mathrm{C} 4, \mathrm{~T} 2-\mathrm{L} 1$ and $\mathrm{S} 3-\mathrm{S} 5)$ the motor level is designated as being the same as the sensory level. In Case 2, the motor level would then be T5. This case therefore aptly illustrates the influence of the neurological level on the AIS. More extensive training in the classification of AIS with the 1996 Standards should help to eliminate this problem.

The results of this study further suggest that training does improve classification of more complicated incomplete injuries. Even the short training session provided in this study resulted in more individuals accurately classifying the injury. With more intensive training and practice, classification should improve. Therefore, it is our recommendation that individuals who classify spinal cord injuries be adequately trained in order to insure accurate classifications. Further, if the information obtained through examination and classification of spinal cord injury is to be entered into a database for research purposes, procedures should be developed for standardized testing of rater reliability for both skills of examination and classification. Such a procedure would insure the consistency of the data from which clinical and research conclusions are drawn. Finally with the clarifications of the neurological classification system, it should be possible to develop a computer algorithm which would classify spinal cord injuries based on the information received from the neurological examination. Our colleagues within the Model Spinal Cord Injury Systems (personal communication $\mathrm{R}$ Marino) are in the process of developing a computer decision tree for the neurological classification of spinal cord injury.

\section{Acknowledgements}

The authors wish to thank Ralph Marino, MD for his insightful comments and review of the manuscript.

\section{References}

1 American Spinal Injury Association (1982) Standard for Neurological Classification of Spinal Injured Patients. ASIA, Chicago

2 Ditunno JF. American Spinal Injury Standards for Neurological and Functional Classification of Spinal Cord Injury: Past, present and future. J Am Paraplegia Soc 1993; 17: 7-11.

3 Donovan WH, et al. A Test of the ASIA Guidelines for Classification of Spinal Cord Injury. J Neuro Rehab 1990; 4: $39-53$.

4 Tator CH et al. (eds) Sunnybrook Cord Injury Scales for Assessing Neurological Injury and Neurological Recovery in Early Management of Acute Spinal Cord Injury 1982; New York, Raven Press.

5 Priebe MM, Waring WP. The interobserver reliability of the revised American Spinal Injury Association Standards for the Neurological Classification of Spinal Injury Patients. Am J Phys Med Rehab 1991; 70: 268-271.

6 Braken MB, et al. A randomized controlled trial of methylprenisolone or naloxone in the treatment of acute spinal cord injury. N Engl J Med 1990; 322: $1405-1411$.

7 Harris P. Editorial: The International Standards Booklet for Neurological and Functional Classification of Spinal Cord Injury. Paraplegia 1994; 32: 69.

8 Ditunno JF, Donovan WH, Maynard FM (eds). Reference Manual for the International Standards for Neurological and Functional Classification of Spinal Cord Injury 1994. ASIA, Chicago. 
ASIA/IMS of standards

ME Cohen et al

9 Cohen ME, Bartko JJ. Reliability of the ISCSCI-92. In: Ditunno JF, Donovan WH, Maynard FM (eds). Reference Manual for the International Standards for Neurological and Functional Classification of Spinal Cord Injury 1994. ASIA, Chicago.

10 Cohen ME, Sheehan TP, Herbison GJ. Content validity and reliability of the International Standards for Neurological Classification of Spinal Cord Injury. Topics in Spinal Cord Injury Rehabilitation 1996; 4: 15-31.
11 American Spinal Injury Association. International Standard for Neurological and Functional Classification of Spinal Cord Injury 1996. ASIA, Chicago.

12 American Spinal Injury Association. Standard for Neurological and Functional Classification of Spinal Cord Injury 1992. ASIA, Chicago. 